\section{Distributed feedback lasers}

from R.G. Harrison

SINCE the gain and so the output power of most gas lasers increases as the inner diameter of the laser tube decreases, considerable attention has been given to the design of laser systems with very narrow bores. For these lasers the electromagnetic resonator modes are altered in a fundamental way from those obtained from conventional open resonator cavities formed by mirrors; the radiation field distribution being no longer determined by the mirrors but by the modes of the hollow waveguide. (Marcatili \& Schmetzer, Bell Syst. Tech. J., 43, 1783; 1964). Laser oscillation is normally sustained in such systems by feedback to the tube from external mirrors. However, an alternative method recently demonstrated is that of distributed feedback (DFB) by which a forward propagating mode is coupled to its corresponding backward mode through scattering from periodic diameter or width changes in the tube. The distinctive feature of this coupling is that the scattered wave excited at a certain position in the waveguide is added in phase to the already existing wave, efficient coupling occurring only when the frequency of the ripple structure in the tube is nearly equal to twice the propagation constant of the lasing mode. Since it is not necessary that this structure be sinusoidal a convenient alternative can be provided by rectangular grooves, coupling then being provided by the fundamental frequency of the periodic rectangular ripple.

For distributed feedback systems the threshold for oscillation increases as the order of resonance of the resonant mode increases. Thus they offer the advantage of operation on the lowest order waveguide mode at a single frequency determined by the resonant mode q; discrimination from other modes being controlled by choosing the gain of the laser such that all higher order modes than the lowest one suffer overall loss. This together with low laser thresholds and the requirement of no external optics makes the technique potentially attractive. Furthermore, the method should not be constrained by the material or cross-sectional geometry of the light tube, and as such it may be applied to many gas laser systems. These include optically pumped lasers for the near to far infrared using either dielectric or metal tubing and also transverse electrically excited high pressure systems and Stark tuned optically pumped lasers, both comprising parallel flat electrodes separated by dielectric spacers to form a rectangular section, metal-dielectric, waveguide.

Distributed feedback laser action was first reported some years back by Kogelnik and Shank (Appl. Phys. Letts., 8, 152; 1971) for a dye system. However it was only recently that such action was achieved in a gas system (Affolter \& Kneubuhl, Phys. Lett., 74A, 407; 1979) even though the theoretical foundations for such operations had been well established (Marcuse, IEEE J. Quantum Electr., QE-10, 413, 1974; Kneubuhl, J. Opt. Soc. $A m ., 11,1 ; 1976)$. Part of the reason lies in matching the laser emission with the periodic structure of the DFB waveguide. For both dye and solid state lasers there is no problem since the emission linewidth are relatively large. However, for both optically pumped and electrically excited gas lasers, usually operated at low pressures, this is not so and matching is correspondingly critical. In the preliminary work of Affolter and Kneubuhl this difficulty has been overcome by temperature tuning the periodic structure of a $\mathrm{CH}_{3} \mathrm{~F}$ optically pumped waveguide system over a temperature range between $30^{\circ} \mathrm{C}$ and $90^{\circ} \mathrm{C}$, the temperature variation of $10^{\circ} \mathrm{C}$ corresponding to a relative change of $\sim 10^{-4}$ of the waveguide period. The bottom of the waveguide was periodically corrugated with rectangular grooves of depth $124 \mu \mathrm{m}$ and period $248 \mu \mathrm{m}$, R.G. Harrison is in the Department of Physics, Heriot-Watt University, Edinburgh.

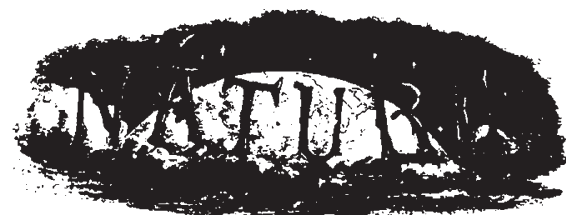

In Nature, vol. xxi. p. III, we described the ingenious planetarium recently invented by Signor Perini, and which has cost him seven years' constant labour. To-day we are able to present an illustration of this invention, which may give those of our readers who have not seen the original, some idea of its construction. The visitors are supposed to be standing underneath the dome, from which 100 years ago

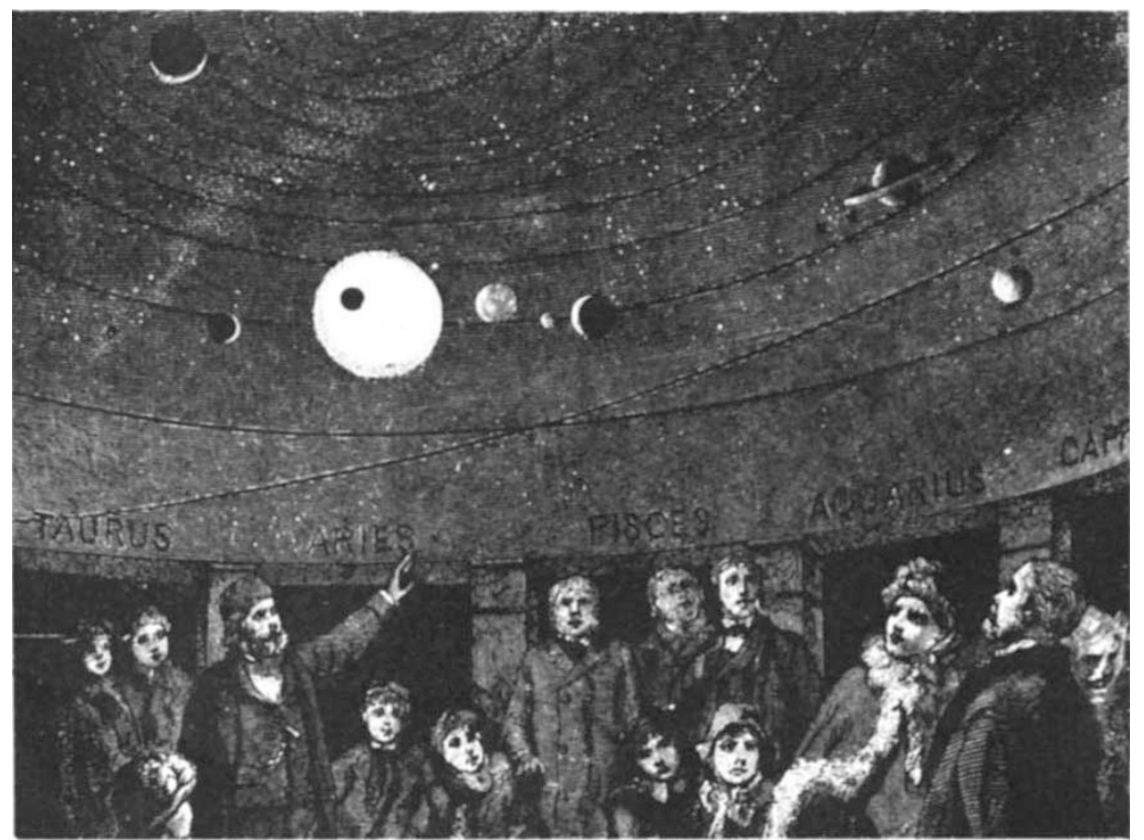

From Nature 586, 15 April, 1880. corresponding to a quarter and a half of the wavelength of the $\mathrm{CH}_{3} \mathrm{~F}$ emission and feedback was optimized by varying the height of the waveguide.

Evidence of DFB action was demonstrated in the resonant or mode $496 \mathrm{~m}$ emission waveguide temperature. Single mode emission was obtained with optimum output at the centre of the temperature at temperatures separated by around $45^{\circ} \mathrm{C}$, corresponding to a mode with DFB theory. The relatively broad temperature bandwidth of around $20^{\circ} \mathrm{C}$ (FWHH) over which oscillation occurs on a single mode, together with the readily accessible temperature range used in the tuning, are both particularly attractive features of the method with regard to control and operation of the system.

Whether the distributed feedback technique will eventually replace cavity optics in some laser ystems remains to be seen. Nevertheless new results are promising and as such effort into the research and development of such systems in the near future. 